\title{
Coronary heart disease incidence and mortality, and all-cause mortality among diabetic and non-diabetic people according to their smoking behavior in Finland
}

Noël C. Barengo ${ }^{1 *}$, Yvonne Teuschl², Vladislav Moltchanov ${ }^{3}$, Tiina Laatikainen ${ }^{3,4,5}$, Pekka Jousilahti ${ }^{4}$ and Jaakko Tuomilehto $2,3,6,7$

\begin{abstract}
Background: As type 2 diabetes (T2D) patients have a high risk for coronary heart disease (CHD) and all-cause mortality and smoking is a major single risk factor for total and CHD mortality, it is important to understand the impact of smoking to the outcome events in comparison to people without T2D. Studies of excess risk of CHD incidence and mortality, and all-cause mortality in T2D patients related to smoking are controversial. We aimed to assess the risk of CHD incidence and mortality, and all-cause mortality in a large Finnish population cohort consisting of people with and without T2Daccording to smoking status.

Methods: Prospective follow-up of 28712 men and 30700 women aged 25-64 years living in eastern and southwestern Finland. The data on mortality were obtained from the nationwide death register using the unique national personal identification number. Follow-up information regarding CHD was based on the Finnish Hospital Discharge Register for non-fatal outcomes. The Cox proportional hazards models were used to estimate the association between diabetes and smoking subgroups and the risk for total and CHD mortality.

Results: T2D patients who were smoking had higher all-cause mortality in both men (HR 3.76; 95\% Cl 2.95-4.78) and women (HR 4.51; 95\% Cl 2.91-7.00) than non-smoking diabetic men (HR 2.03; 95\% Cl 1.51-2.74) and women (HR 2.11; 95\% Cl 1.71-2.59). The CHD mortality risk for smoking men with T2D was higher (HR 6.15; 95\% Cl 4.22-8.96) than in non-smoking diabetic men (HR 2.62; 95\% Cl 1.60-4.29). Similar results were found in women revealing corresponding HR for CHD mortality of 6.92 (95\% Cl 2.79-17.19) for smoking, T2D women and 4.06 (95\% Cl 2.83-5.82) for non-smoking T2D women, respectively. Even though the risk of CHD incidence in T2D patients who had stopped smoking was statistically significantly higher than in their non-smoking non-diabetic counterparts, their CHD incidence was lower than in smoking T2D patients (HR in men 3.00; HR in women 2.80).
\end{abstract}

Conclusion: It is important to address tobacco consumption in T2D patients, especially during primary health care contacts in order to reduce their high risk of CHD and all-cause mortality.

Keywords: Smoking, Diabetes mellitus, Mortality, Coronary heart disease

\footnotetext{
* Correspondence: nbarengo@fiu.edu

${ }^{1}$ Department of Medical and Population Health Sciences Research, Herberth Wertheim College of Medicine, Florida International University, 11200 SW 8th Street, AHC2, Miami, FL 33199, USA

Full list of author information is available at the end of the article
} 


\section{Background}

Type 2 diabetes (T2D) is one of the fastest growing public health problems in both developed and developing countries imposing a high financial burden on health care costs. The IDF has estimated that the numbers of adults with diabetes is expected to rise from 415 million in 2015 to 642 million by 2040 [1]. Most people with diabetes will sooner or later develop cardiovascular diseases (CVD) [2]. People with T2D have a higher risk of CVD and all-cause mortality than the rest of the population and once developing CVD their prognosis are poorer [3, 4]. Premature mortality caused by diabetes results in an estimated 12-14 years of life lost [5].

Studies of excess risk of coronary heart disease (CHD) and all-cause mortality in T2D patients related to smoking are controversial. This is surprising as smoking is one of the classical risk factors for CVD besides hypertension, hyperlipidaemia and diabetes, and contributes substantially to the global burden of disease $[6,7]$. While some studies observed an additive increased risk of CHD and all-cause mortality [8-12], others did not find that smoking amplifies the risk of CHD or total mortality rates among $\mathrm{T} 2 \mathrm{D}$ patients [13-17]. It has been argued that these conflicts may be attributed to low power or inadequate adjustment for confounders [18].

As T2D patients have shown to have a high risk for CHD and all-cause mortality and smoking is a major single risk factor for total and CHD mortality, it is important to understand the impact of smoking to these outcome events in people with T2D in comparison with people without T2D.

The aim of this study was to assess the risks of CHD and all-cause mortality in a large Finnish population cohort consisting of people with and without diabetes according to smoking status.

\section{Methods}

\section{Study population}

Five independent cross-sectional surveys were carried out at five-year intervals within the framework of the FINMONICA/FINRISK studies [19] between 1982 and 2002. An independent random sample was drawn from the national population register for each survey. The samples were stratified by sex and 10-year age categories according to the WHO MONICA protocol so that at least 250 people of each sex and 10-year age group were chosen [20]. The study participants included in the five surveys were 25 to 64 years of age, and the 1997 and 2002 surveys also included subjects aged 65 to 74 years. The participation rate varied in the survey cohorts between $63 \%$ and $83 \%$ in men and between $72 \%$ and $88 \%$ in women. The final sample comprised 28 712 men and 30700 women.

\section{Baseline data collection}

A self-administered questionnaire was mailed to the participants in advance. The questionnaire included questions on health behavior, such as smoking habits, and physical activity, education and medical history. Smoking habits were classified to three categories: never smokers, ex-smokers (those, who had smoked regularly but had stopped smoking at least six month before the survey) and current smokers.

The participants reported their occupational and leisure time physical activity. These were merged and regrouped into three categories (low, moderate and high) of total physical activity as described in previous publications [21, 22]. Education level, measured as the total number of school years, was divided into birth cohort-specific tertiles.

At the study site, specially trained nurses measured height, weight, and blood pressure (BP) using a standardized protocol [20]. Height and weight were measured without shoes and with light clothing. BP was measured twice from the right arm of the participant in sitting position after at least 5 min rest. A standard mercury sphygmomanometer was used. Starting from 1982, BP was measured twice and the mean of these two BP measurements was used in the analyses.

Serum total cholesterol was determined by using an enzymatic method (CHOD-PAP, Boehringer Mannheim, Mannheim, Germany). All samples were analyzed in the biochemical laboratory of the National Public Health Institute (nowadays National Institute for Health and Welfare), which is an accredited laboratory.

Participants who reported having diabetes on the questionnaire, or who had had a hospital discharge diagnosis of diabetes or the approval for free-of-charge medication for diabetes before the baseline survey were classified as having diabetes. Data on diabetes medication were ascertained from the national Social Insurance Institution's register on special reimbursement for anti-diabetic drugs from 1964. Anti-diabetic drugs prescribed by a physician are reimbursed by social insurance in Finland and are subject to approval of a physician of the Institution who reviews each case history.

\section{CHD incidence and mortality}

The original survey data were complemented by linkage to the nationwide death register of Statistics Finland according the unique national personal identification number that every Finnish resident has. These records covered the period from January 1970 to December 2008. The Eighth, Ninth and Tenth Revisions of the International Classification of Diseases (ICD) were used for coding the causes of death. The end point of the follow-up was the date of death. 
Follow-up information regarding CHD was based on the Finnish Hospital Discharge Register for non-fatal outcomes. The ICD codes used for CHD incidence and mortality were as follows: non-fatal myocardial infarction 410-411 (ICD9) and I21-I22 (ICD10) and fatal cases of CHD 410-414 (ICD9) and I20-I25 (ICD10). The end points during follow-up was incident CHD, defined as either the first non-fatal CHD or CHD death. The calendar period of follow up was truncated by the same date, 31/12/2008, as death information.

\section{Statistical analysis}

Statistical analyses were performed using SAS 9.2. The Cox proportional hazards models were used to estimate the association between diabetes and smoking subgroups and the risk for total and CHD mortality. Analyses were adjusted first for age, study area and study year (A) then for age, study region, study year, education, systolic blood pressure (SBP), cholesterol level, physical activity, alcohol consumption and body mass index (BMI) (B). The data of all six surveys were pooled together as no first level interaction were found between diabetes/ smoking groups and these variables regarding $\mathrm{CHD}$, stroke or total mortality, respectively incidence. The proportional hazards assumption in the Cox model was tested based on the Schoenfeld Residuals. Estimated hazard ratios (HR) and their 95\% confidence intervals (CI) are presented.

\section{Results}

The baseline characteristics of the study participants are presented in Table 1 according to sex and smoking status. Among men with T2D 32\% were current and 37\% ex-smokers. The corresponding prevalence of smoking

Table 1 Baseline characteristics of the study population according to diabetes and smoking status

\begin{tabular}{|c|c|c|c|c|c|c|}
\hline & \multicolumn{3}{|l|}{ No diabetes } & \multicolumn{3}{|l|}{ Diabetes } \\
\hline & Non-smokers & Ex-smokers $^{a}$ & Smokers $^{\mathrm{b}}$ & Non-smokers & Ex-smokers & Smokers \\
\hline Men (n) & 9539 & 7063 & 11054 & 323 & 392 & 341 \\
\hline Age (years) & $43.2(11.9)^{c}$ & $48.5(11.7)$ & $42.6(11.1)$ & $54.1(11.5)$ & $56.9(9.1)$ & $51.0(10.4)$ \\
\hline \multicolumn{7}{|l|}{ Education (\%) } \\
\hline Low & 28 & 29 & 37 & 31 & 31 & 33 \\
\hline Moderate & 33 & 37 & 35 & 31 & 35 & 34 \\
\hline High & 39 & 34 & 28 & 38 & 34 & 33 \\
\hline Body mass index $\left(\mathrm{kg} / \mathrm{m}^{2}\right)$ & $26.1(3.5)$ & $27.3(3.7)$ & $25.8(3.7)$ & $28.5(4.4)$ & $29.3(4.0)$ & $27.8(4.9)$ \\
\hline Systolic blood pressure $(\mathrm{mmHg})$ & $140(18)$ & $144(20)$ & $142(19)$ & $148(24)$ & $148(21)$ & $146(21)$ \\
\hline Serum cholesterol $(\mathrm{mmol} / \mathrm{l})$ & $5.8(1.2)$ & $6.1(1.2)$ & $6.2(1.31)$ & $5.7(1.2)$ & $5.9(1.3)$ & $6.1(1.4)$ \\
\hline \multicolumn{7}{|l|}{ Total physical activity (\%) } \\
\hline Low & 8 & 10 & 13 & 16 & 21 & 24 \\
\hline Moderate & 83 & 84 & 82 & 81 & 77 & 75 \\
\hline High & 9 & 6 & 5 & 3 & 2 & 1 \\
\hline Women (n) & 21463 & 2980 & 5101 & 878 & 125 & 153 \\
\hline Age (years) & $45.7(11.6)$ & $42.5(11.6)$ & $40.4(10.8)$ & $54.0(11.1)$ & $51.2(11.9)$ & $46.7(11.0)$ \\
\hline \multicolumn{7}{|l|}{ Education (\%) } \\
\hline Low & 32 & 31 & 40 & 36 & 31 & 38 \\
\hline Moderate & 34 & 33 & 33 & 34 & 39 & 32 \\
\hline High & 34 & 36 & 27 & 30 & 30 & 30 \\
\hline Body mass index $\left(\mathrm{kg} / \mathrm{m}^{2}\right)$ & $26.2(4.8)$ & $25.9(4.6)$ & $24.9(4.5)$ & $29.7(6.1)$ & $31.1(6.3)$ & $28.4(5.4)$ \\
\hline Systolic blood pressure $(\mathrm{mmHg})$ & $140(23)$ & $131(19)$ & $131(19)$ & $148(24)$ & $145(23)$ & $138(23)$ \\
\hline Serum cholesterol (mmol/l) & $6.0(1.3)$ & $5.5(1.2)$ & $5.6(1.2)$ & $6.0(1.3)$ & $5.7(1.1)$ & $6.0(1.3)$ \\
\hline \multicolumn{7}{|l|}{ Total physical activity (\%) } \\
\hline Low & 15 & 13 & 17 & 27 & 29 & 28 \\
\hline Moderate & 82 & 83 & 80 & 71 & 70 & 70 \\
\hline High & 3 & 4 & 3 & 2 & 1 & 2 \\
\hline
\end{tabular}

athose, who had smoked regularly but had stopped smoking at least six month before the survey

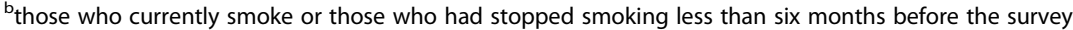

${ }^{c}$ mean and standard deviation 
and ex-smoking in men without T2D was $40 \%$ and $26 \%$, respectively. Among women with T2D 13\% were current and $11 \%$ ex-smokers. The corresponding prevalence of smoking and ex-smoking in women without T2D was $17 \%$ and $10 \%$, respectively. People with T2D seemed to have a higher prevalence of overweight and obesity, lower levels of physical activity, higher serum cholesterol and systolic blood pressure level than people without diabetes at baseline or during follow-up. This pattern was observed in both men and women.

\section{T2D, smoking and all-cause mortality}

The HRs for all-cause mortality were higher for T2D patients compared with men and women without T2D. T2D patients who were smoking had higher all-cause mortality in both men (HR 3.76; 95\% CI 2.95-4.78) and women (HR 4.51; 95\% CI 2.91-7.00) than non-smoking diabetic men (HR 2.03; 95\% CI 1.51-2.74) and women (HR 2.11; 95\% CI 1.71-2.59) (Table 2). T2D patients that had stopped smoking also had increased all-cause mortality compared with non-smoking, diabetic men and women when adjusted for age, study area and study year, education, systolic blood pressure, physical activity, serum cholesterol and BMI (men: HR 2.56; 95\% CI 2.043.22), women: HR 2.61; 95\% CI 1.57-4.31).

\section{T2D, smoking and CHD mortality}

Table 3 shows the hazard ratios for CHD mortality among men and women with and without diabetes according to smoking status. The CHD mortality risk for smoking men with T2D was higher (HR 6.15; 95\% CI 4.22-8.96) than in non-smoking diabetic men (HR 2.62; 95\% CI 1.60-4.29). Similar results were found in women revealing corresponding HR for CHD mortality of $6.92(95 \% \mathrm{CI}$ 2.79-17.19) for smoking, T2D women and 4.06 (95\% CI 2.83-5.82) for non-smoking T2D women, respectively. T2D patients that had stopped smoking also had a higher risk of CHD mortality compared with non-smoking, diabetic men and women when adjusted for age, study area and study year, education, systolic blood pressure, physical activity, serum cholesterol and BMI (men: HR 4.30; 95\% CI 3.04-6.08), women: HR 5.00; 95\% CI 2.15-11.64). The HRs of CHD mortality in smoke-free T2D patients was statistically significantly higher compared with non-smoking people without T2D (men: HR 2.62; 95\% CI 1.60-4.29; women: HR 4.06; 95\% CI 2.83-5.82).

\section{T2D, smoking and CHD incidence}

Smoking T2D patients had an increase in CHD incidence compared with non-smoking people with and without T2D (Table 4). The risk increase in smoking diabetic men was 3.27-fold (95\% CI 2.45-2.40) and 4.55-fold in women (95\% CI 2.48-8.33) when controlled for age, education, systolic blood pressure, physical activity, serum cholesterol and BMI. Even though the risk of CHD incidence in T2D patients who had stopped smoking was statistically significantly higher than in their non-smoking non-diabetic

Table 2 Hazard Ratio (HR) of all-cause mortality among men and women with and without diabetes at baseline according to smoking status

\begin{tabular}{|c|c|c|c|c|c|}
\hline & \multicolumn{3}{|l|}{ Model $A^{b}$} & \multicolumn{2}{|c|}{ Model $B^{C}$} \\
\hline & Mortality rate $^{a}$ & $\mathrm{HR}$ & $95 \% \mathrm{Cl}$ & $\overline{\mathrm{HR}}$ & $95 \% \mathrm{Cl}$ \\
\hline \multicolumn{6}{|l|}{ Men } \\
\hline Non-diabetic, non-smokers & 0.88 & 1 & $\operatorname{Ref}^{d}$ & 1 & Ref \\
\hline Non-diabetic, ex-smokers & 1.52 & 1.46 & $1.29-1.66$ & 1.38 & $1.21-1.57$ \\
\hline Non-diabetic, smokers & 1.93 & 2.98 & $2.65-3.35$ & 2.63 & $2.33-2.97$ \\
\hline Diabetic, non-smokers & 2.63 & 2.22 & $1.66-2.95$ & 2.03 & $1.51-2.74$ \\
\hline Diabetic, ex-smokers & 3.30 & 2.94 & $2.35-3.67$ & 2.56 & $2.04-3.22$ \\
\hline Diabetic, smokers & 3.97 & 4.35 & $3.44-5.51$ & 3.76 & $2.95-4.78$ \\
\hline \multicolumn{6}{|l|}{ Women } \\
\hline Non-diabetic, non-smokers & 0.92 & 1 & Ref & 1 & Ref \\
\hline Non-diabetic, ex-smokers & 0.44 & 1.27 & $1.02-1.59$ & 1.30 & $1.04-1.63$ \\
\hline Non-diabetic, smokers & 0.85 & 2.41 & $2.06-2.81$ & 2.37 & $2.02-2.78$ \\
\hline Diabetic, non-smokers & 2.43 & 2.40 & $1.96-2.94$ & 2.11 & $1.71-2.59$ \\
\hline Diabetic, ex-smokers & 1.75 & 3.25 & $1.98-5.36$ & 2.61 & $1.57-4.31$ \\
\hline Diabetic, smokers & 1.71 & 4.96 & $3.21-7.67$ & 4.51 & $2.91-7.00$ \\
\hline
\end{tabular}

anumber of events per 100 person-years

badjusted for age, study area and study year

cadjusted for age, study area, study year, education, systolic blood pressure, physical activity, serum cholesterol and BMI

${ }^{d}$ reference group 
Table 3 Hazard Ratio (HR) of coronary heart disease (CHD) mortality among men and women with and without diabetes at baseline according to smoking status

\begin{tabular}{|c|c|c|c|c|c|}
\hline & \multicolumn{3}{|l|}{ Model $A^{b}$} & \multicolumn{2}{|c|}{ Model BC } \\
\hline & Mortality rate ${ }^{a}$ & $\mathrm{HR}$ & $95 \% \mathrm{Cl}$ & $\overline{\mathrm{HR}}$ & $95 \% \mathrm{Cl}$ \\
\hline \multicolumn{6}{|l|}{ Men } \\
\hline Non-diabetic, non-smokers & 0.29 & 1 & $\operatorname{Ref}^{d}$ & 1 & Ref \\
\hline Non-diabetic, ex-smokers & 0.56 & 1.71 & $1.36-2.16$ & 1.59 & $1.25-2.02$ \\
\hline Non-diabetic, smokers & 0.65 & 3.29 & $2.64-4.10$ & 3.11 & $2.48-3.92$ \\
\hline Diabetic, non-smokers & 1.06 & 2.86 & $1.77-4.61$ & 2.62 & $1.60-4.29$ \\
\hline Diabetic, ex-smokers & 1.63 & 5.28 & $3.77-7.40$ & 4.30 & $3.04-6.08$ \\
\hline Diabetic, smokers & 1.73 & 6.98 & $4.85-10.03$ & 6.15 & $4.22-8.96$ \\
\hline \multicolumn{6}{|l|}{ Women } \\
\hline Non-diabetic, non-smokers & 0.25 & 1 & Ref & 1 & Ref \\
\hline Non-diabetic, ex-smokers & 0.08 & 1.33 & $0.74-2.37$ & 1.40 & $0.78-2.51$ \\
\hline Non-diabetic, smokers & 0.20 & 3.43 & $2.40-4.89$ & 3.84 & $2.65-5.55$ \\
\hline Diabetic, non-smokers & 0.96 & 4.81 & $3.37-6.85$ & 4.06 & $2.83-5.82$ \\
\hline Diabetic, ex-smokers & 0.75 & 7.73 & $3.37-17.73$ & 5.00 & $2.15-11.64$ \\
\hline Diabetic, smokers & 0.57 & 8.79 & $3.56-21.67$ & 6.92 & $2.79-17.19$ \\
\hline
\end{tabular}

${ }^{a}$ number of events per 100 person-years

badjusted for age, study area and study year

cadjusted for age, study area, study year, education, systolic blood pressure, physical activity, serum cholesterol and BMI

dreference group

Table 4 Hazard Ratio (HR) of coronary heart disease incidence among men and women with and without diabetes at baseline according to smoking status

\begin{tabular}{|c|c|c|c|c|c|}
\hline & \multicolumn{3}{|l|}{ Model $A^{b}$} & \multicolumn{2}{|c|}{ Model $B^{C}$} \\
\hline & Incidence rate ${ }^{a}$ & $H R$ & $95 \% \mathrm{Cl}$ & $\mathrm{HR}$ & $95 \% \mathrm{Cl}$ \\
\hline \multicolumn{6}{|l|}{ Men } \\
\hline Non-diabetic, non-smokers & 0.69 & 1 & $\operatorname{Ref}^{d}$ & 1 & Ref \\
\hline Non-diabetic, ex-smokers & 1.10 & 1.25 & $1.08-1.44$ & 1.15 & $0.99-1.32$ \\
\hline Non-diabetic, smokers & 1.21 & 2.04 & $1.79-2.33$ & 1.95 & $1.70-2.23$ \\
\hline Diabetic, non-smokers & 1.85 & 1.70 & $1.19-2.44$ & 1.56 & $1.08-2.24$ \\
\hline Diabetic, ex-smokers & 2.98 & 3.66 & $2.87-4.68$ & 3.00 & 2.33-3.85 \\
\hline Diabetic, smokers & 2.69 & 3.51 & $2.64-4.67$ & 3.27 & $2.45-4.36$ \\
\hline \multicolumn{6}{|l|}{ Women } \\
\hline Non-diabetic, non-smokers & 0.50 & 1 & Ref & 1 & Ref \\
\hline Non-diabetic, ex-smokers & 0.19 & 1.21 & $0.88-1.66$ & 1.27 & $0.92-1.74$ \\
\hline Non-diabetic, smokers & 0.39 & 2.13 & $1.70-2.67$ & 2.32 & $1.84-2.92$ \\
\hline Diabetic, non-smokers & 1.52 & 2.94 & $2.30-3.77$ & 2.60 & $2.02-3.35$ \\
\hline Diabetic, ex-smokers & 0.99 & 3.59 & $1.91-6.76$ & 2.80 & $1.48-5.30$ \\
\hline Diabetic, smokers & 1.24 & 5.37 & $3.02-9.58$ & 4.55 & $2.48-8.33$ \\
\hline
\end{tabular}

${ }^{a}$ number of events per 100 person-years

badjusted for age, study area and study year

cadjusted for age, study area, study year, education, systolic blood pressure, physical activity, serum cholesterol and BMI

${ }^{d}$ reference group 
counterparts, the CHD incidence was lower than in smoking T2D patients (HR in men 3.00; HR in women 2.80).

\section{Discussion}

This study showed that smoking men and women with T2D had a higher risk of CHD incidence, and CHD and all-cause mortality compared with non-smoking people free of diabetes. Stopping smoking reduced the risk of allcause mortality in both men and women with T2D. Whereas T2D patients who were not smoking or who had stopped smoking had an increased risk of CHD incidence and mortality, that increase seemed to be lower than the one observed in their smoking diabetic counterparts.

As smoking may influence various mechanisms proposed to increase the risk of $\mathrm{CHD}$ (e.g. vascular endothelial injury, increased oxidative stress, thromboembolism), it is possible that the effect of smoking on CHD and MI might be more dramatically increased in diabetic patients, by a combination of short-term effects (coronary artery spasm, arrhythmias) and long-term effects (metabolic and thrombotic disorders) on the cardiovascular system [23, 24]. In T2D patients, smoking is an independent risk factor for $\mathrm{CHD}$, stroke and peripheral vascular disease [8, 25-27]. It accentuates the dyslipidemia of T2D that is associated with increased hepatic lipase activity that may produce atherogenic, small, dense LDL particles [28].

In agreement with our study, the Nurses' Health Study showed that the age-adjusted incidence rate of total CHD was much higher among diabetic women than among non-diabetic women of similar smoking status. However, they observed a considerably higher hazard ratio for CHD among diabetic women than for non-diabetic women of similar smoking status compared with our study [8] presenting a multivariate adjusted HR 7.7 for CHD in women with T2D smoking more than 15 cigarettes/day compared with non-diabetic, nonsmoking women [8]. Furthermore, current smokers seem to be at higher risk than exsmokers in regard all-cause and CHD mortality. These findings are in agreement with previous studies showing that previous smokers might still suffer from the adverse effects related to smoking even though the risk started to decrease [8-10].

Most studies have compared mortality rates according to smoking status among patients with T2D rather than comparing mortality rates with the general population [7, 9-11]. A cohort study involving 13087 female and male patients with T2D from the Swedish National Diabetes Register revealed statistically significant hazard ratios for smoking and first-incident fatal/nonfatal MI and total mortality of 1.7 and 1.8, respectively [7]. The Nurses' Health Study reported that cigarette smoking was associated in a dose-response manner with an increased mortality among women with type 2 diabetes [9] and the United Kingdom prospective diabetes study found that hazard ratio for smokers in regard CHD of 1.41 (1.06 to 1.88) among a 7.9 year follow-up of 3055 T2D patients (10). Finally, a three year follow-up of 390 elderly T2D patients (mean age 73 years) showed an increased the risk of mortality 2.58 (95\% CI 1.30-5.11) among the smoking T2D patients compared with the non-smoking ones [11].

Interestingly, some cohort studies did not find that smoking increased the risk of CHD or all-cause mortality among T2D patients [12-17]. The Aerobics Center Longitudinal Study included 2316 men with T2D which were followed-up for almost 16 years [12] and the Rochester Epidemiology Project comprised 337 T2D patients of Olmsted County, Minnesota [17]. Even though both studies reported increased risks for current and exsmoking in regard CVD or all-cause mortality, this risk increase did not reach statistical significance. Another prospective population-based study of 400 patients with T2D in Skara, Sweden revealed a 1.66-fold risk increase for all-cause mortality among smokers compared with non-smokers with a 95\% CI of 0.99-2.76 [14]. Probably, the latter two studies may have a low power to detect risk increases due to a small sample size [14, 17]. Furthermore, both the ZODIAC-10 study and the Milan Study on Atherosclerosis and Diabetes were lacking adequate adjustments of their results $[15,16,18]$.

A recent meta-analysis of observational prospective studies assessing the excess risk of mortality and CVD events associated with smoking among patients with diabetes revealed a relative risk comparing smokers with nonsmokers of 1.48 for total mortality 1.54 for CHD [18]. Furthermore, that excess risk was observed among former and current smokers with a greater risk in current smokers.

The life-extending effect of successful smoking cessation ultimately concerns every smoking patient in medical practice and physicians should have an ethical obligation to educate their patients about smoking and should not hesitate to routinely advise to quit [29, 30]. In Finland, unfortunately, smoking is not discussed during most routine health care consultations with smokers if they do not present any "smoking-related" disease [31]. Physicians should ask their patients whether they smoke, how much they smoke, and whether they are willing to quit. As a subsequent step one should explain to every smoking patient why and to what extent smoking cessation would, for medical reasons, favorably change their future health. This short talk hardly takes more than two minutes [29]. It has been shown that if the patient presents CHD, approximately eight out of ten Finnish primary care physicians give smoking cessation advice to that specific population group [31]. However, smoking counseling should be given long before the patients have CHD and especially if they have T2D as pointed out in the current clinical guidelines for diabetes care [32]. 
Naturally, our study had some limitations. The baseline assessment of our cohort is limited to the examination on a single day when participants entered the study, as typical for large cohort studies. It cannot account for changes in lifestyle or risk factors of CHD disease leading to a shift of participants between categories during the study period. Furthermore, we cannot completely exclude the effects of confounding due to some unmeasured dietary and other lifestyle factors that may influence CHD or all-cause mortality. In addition, assessing smoking habits by questionnaire may lack some of accuracy as we did not have information regarding packyears or intensity of smoking. However, self-reported smoking habits are commonly used in epidemiological studies and seem to be rather accurate when compared with biochemical markers of tobacco use.

\section{Conclusions}

It is important to address tobacco consumption in T2D patients in primary health-care in order to reduce their risk of CHD and all-cause mortality. Smoking cessation programs should be offered to each T2D patient and former smoking T2D patients should be encouraged to remain smoke-free. As smoking and ex-smoking T2D patients have a higher risk of CHD incidence and mortality, their health status with regard to CHD should be carefully checked during their annual follow-up control visits with health care personnel.

\section{Acknowledgements}

Not applicable

\section{Funding}

This work was supported by a grant of the Suomen Lääketieteen Säätiö.

\section{Availability of data and materials}

Please contact author for data requests.

\section{Authors' contributions}

NCB and YT planned the study. VM, NCB and JT were responsible for study planning and data analysis. All authors reviewed the results of the data analysis, report and draft manuscript. All authors have read and approved the final version of the manuscript.

\section{Competing interests}

The authors declare that they have no competing interests.

\section{Consent for publication}

Not applicable.

\section{Ethics approval and consent to participate}

This study followed the Good Clinical Practice guidelines and the guidelines of the Helsinki Declaration. An ethical approval has been obtained for each survey round from the ethics committee valid at the time. Informed written content was obtained from all participants starting from the 1997 survey. At the time of the earlier survey a written consent was not asked, but the participants were informed about the purpose of the study both in written and oral form.

\section{Author details}

${ }^{1}$ Department of Medical and Population Health Sciences Research, Herberth Wertheim College of Medicine, Florida International University, 11200 SW 8th
Street, AHC2, Miami, FL 33199, USA. ²Department of Neurosciences and Preventive Medicine, Danube-University Krems, Krems, Austria. ${ }^{3}$ Department of Health, National Institute for Health and Welfare, Helsinki, Finland. ${ }^{4}$ Institute of Public Health and Clinical Nutrition, University of Eastern Finland, Kuopio, Finland. ${ }^{5}$ Hospital District of North Karelia, Joensuu, Finland. ${ }^{6}$ Dasman Diabetes Institute, Dasman, Kuwait. ${ }^{7}$ Diabetes Research Group, King Abdulaziz University, Jeddah, Saudi Arabia.

Received: 12 April 2016 Accepted: 12 January 2017

Published online: 02 February 2017

\section{References}

1. International Diabetes Federation (IDF). Diabetes atlas. 7th ed. Brussels: IDF; 2015.

2. The DECODE Study Group. Is the current definition for diabetes relevant to mortality risk from All causes and cardiovascular and noncardiovascular diseasese. Diabetes Care. 2003;26:688-99.

3. Barengo NC, Katoh S, Moltchanov S, Tajima N, Tuomilehto J. The diabetescardiovascular risk paradox: results from a Finnish population-based prospective study. Eur Heart J. 2008;29(15):1889-95.

4. Laukkanen JA, Mäkikallio TH, Ronkainen K, Karppi J, Kurl S. Impaired fasting plasma glucose and type 2 diabetes Are related to the risk of Out-ofhospital sudden cardiac death and All-cause mortality. Diabetes Care. 2013; 36(5):1166-71

5. Manuel D, Schultz S. Health-related quality of life and health-adjusted life expectancy of people with diabetes mellitus in Ontario, Canada, 1996-1997. Diabetes Care. 2004;27:407-14.

6. Murray CJ, Lopez AD. Global mortality, disability, and the contribution of risk factors: global burden of disease study. Lancet. 1997;349:1436-42.

7. Yusuf S, Hawken S, Ounpuu S, Dans T, Avezum A, Lanas F, et al. INTERHEART study investigators. Effect of potentially modifiable risk factors associated with myocardial infarction in 52 countries (the INTERHEART study): casecontrol study. Lancet. 2004;364:937-52.

8. Nilsson PM, Cederholm J, Eeg-Olofsson K, et al. Smoking as an independent risk factor for myocardial infarction or stroke in type 2 diabetes: a report from the swedish national diabetes register. Eur J Cardiovasc Prev Rehabil. 2009;16:506-12.

9. Al-Delaimy WK, Manson JE, Solomon CG, et al. Smoking and risk of coronary heart disease among women with type 2 diabetes mellitus. Arch Intern Med. 2002;162:273-9.

10. Al-Delaimy WK, Willett WC, Manson JE, Speizer FE, Hu FB. Smoking and mortality among women with type 2 diabetes: the Nurses' health study cohort. Diabetes Care. 2001;24:2043-8.

11. Turner RC, Millns H, Neil HAW, Stratton IM, Manley SE, Matthews DR, et al. Risk factors for coronary artery disease in non-insulin dependent diabetes mellitus: united kingdom prospective diabetes study (UKPDS: 23). BMJ. 1998;316:823-8.

12. Katakura $M$, Naka M, Kondo T, et al. Prospective analysis ofmortality, morbidity, and risk factors in elderly diabetic subjects: Nagano study. Diabetes Care. 2003;26:638-44

13. Church TS, LaMonte MJ, Barlow CE, Blair SN. Cardiorespiratory fitness and body mass index as predictors of cardiovascular disease mortality among men with diabetes. Arch Intern Med. 2005;165:2114-20.

14. Ostgren CJ, Lindblad U, Melander A, Rastam L. Survival in patients with type 2 diabetes in a swedish community: skaraborg hypertension and diabetes project. Diabetes Care. 2002;25:1297-302.

15. Faglia E, Favales F, Calia P, et al. Cardiac events in 735 type 2 diabetic patients who underwent screening for unknown asymptomatic coronary heart disease: 5-year follow-up report from the Milan study on atherosclerosis and diabetes (MiSAD). Diabetes Care. 2002;25:2032-6.

16. Lutgers HL, Gerrits EG, Sluiter WJ, et al. Life expectancy in a large cohort of type 2 diabetes patients treated in primary care (ZODIAC-10). PLoS One. 2009;4(8):e6817.

17. Adams LA, Harmsen S, St. Sauver JL, et al. Nonalcoholic fatty liver disease increases risk of death among patients with diabetes: a community-based cohort study. Am J Gastroenterol. 2010;105:1567-73.

18. Qin R, Chen T, Lou Q, Yu D. Excess risk of mortality and cardiovascular events associated with smoking among patients with diabetes: meta-analysis of observational prospective studies. Int J Cardiol. 2013:167(2):342-50.

19. Vartiainen E, Jousilahti P, Alfthan G, Sundvall J, Pietinen P, Puska P. Cardiovascular risk factor changes in Finland, 1972-1997. Int J Epidemiol. 2000;29(1):49-56. 
20. The World Health Organization MONICA Project (monitoring trends and determinants in cardiovascular disease). a major international collaboration. WHO MONICA project principal investigators. J Clin Epidemiol. 1988;41(2):105-14.

21. Hu G, Jousilahti P, Barengo NC, Qiao Q, Lakka TA, Tuomilehto J. Physical activity, cardiovascular risk factors, and mortality among Finnish adults with diabetes. Diabetes Care. 2005;28:799-805.

22. Hu G, Tuomilehto J, Silventoinen K, Barengo NC, Jousilahti P. The effects of physical activity and body mass index on cardiovascular, cancer and allcause mortality among 47212 middle-aged Finnish men and women. Int J Obes Relat Metab Disord. 2005;29:894-902.

23. Pyorala K, Laakso M, Uusitupa M. Diabetes and atherosclerosis: an epidemiologic view. Diabetes Metab Rev. 1987;3:463-524.

24. Facchini F, Hollenbeck C, Jeppesen J, Chen Y, Reaven G. Insulin resistance and cigarette smoking. Lancet. 1992;339:1128-30.

25. Walters DP, Gatling W, Houston AC, Mullee MA, Julious SA, Hill RD. Mortality in diabetic subjects: an eleven-year follow-up of a community-based population. Diabetic Med. 1994;11:968-73.

26. Kothari V, Stevens RJ, Adler Al, Stratton IM, Manley SE, Neil HA, et al. UKPDS 60: risk of stroke in type 2 diabetes estimated by the UK prospective diabetes study risk engine. Stroke. 2002;33:1776-81.

27. Adler Al, Stevens RJ, Neil A, Stratton IM, Boulton AJ, Holman RR. UKPDS 59 hyperglycemia and other potentially modifiable risk factors for peripheral vascular disease in type 2 diabetes. Diabetes Care. 2002;25:894-9.

28. Kong C, Nimmo L, Elatrozy T, Anyaoku V, Hughes C, Robinson S, et al. Smoking is associated with increased hepatic lipase activity, insulin resistance, dyslipidaemia and early atherosclerosis in type 2 diabetes. Atherosclerosis. 2001;156:373-8.

29. Eckert T, Junker C. Motivation for smoking cessation: what role do doctors play? Swiss Med Wkly. 2001;131:521-6.

30. Liu JL, Tang J-L. Doctors are ethically obliged to advise patients to quit smoking. BMJ. 1998;317:1588

31. Barengo NC, Sandström HP, Jormanainen VJ, Myllykangas MT. Attitudes and behaviour in smoking cessation among general practitioners in Finland. Soz Praventivmed. 2005;50(6):355-60.

32. Ryden L, Standl E, Bartnik M, Van den Berghe G, Betteridge J, de Boer MJ, et al. Guidelines on diabetes, pre-diabetes, and cardiovascular diseases: executive summary. The task force on diabetes and cardiovascular diseases of the european society of cardiology (ESC) and of the european association for the study of diabetes (EASD). Eur Heart J. 2007:28:88-136.

\section{Submit your next manuscript to BioMed Central and we will help you at every step:}

- We accept pre-submission inquiries

- Our selector tool helps you to find the most relevant journal

- We provide round the clock customer support

- Convenient online submission

- Thorough peer review

- Inclusion in PubMed and all major indexing services

- Maximum visibility for your research

Submit your manuscript at www.biomedcentral.com/submit

) Biomed Central 